\title{
Use of ultrasonography as a noninvasive decisive tool to determine the accurate endotracheal tube size in anesthetized children
}

\author{
Gulnur Gollu, Assist Prof.; A. Onat Bermede, M.D. ; Farid Khanmammadov, M.D.a; \\ Ufuk Ates, M.D. ${ }^{a}$; Sinan Genc, M.D. .; Ozlem Selvi Can, Assoc. Prof. ; \\ Suat Fitoz, Prof.; Zekeriyya Alanoglu, Prof. ${ }^{b}$ and Aydin Yagmurlu, Prof. ${ }^{a}$
}

\begin{abstract}
Background. It is hard to determine the appropriate size and correct tracheal position of endotracheal tube (ETT) in children. The aim of this study is to determine tracheal diameter in children by using ultrasonography technique as objective tool and compare it with commonly used aged based formulas for the ETT size estimation.

Patients and methods. Patients undergoing elective surgery in a tertiary children's hospital were prospectively enrolled. The subglottic transverse tracheal diameter was determined by ultrasonography. An anesthesiologist who was blind to ultrasonographic examination, determined the tube size and performed intubation by evaluating the space between vocal cords with the help of a direct laryngoscopic view. Ultrasonographically measured tracheal diameter, tubediameters, leak/ pressure controls, and results of age-based tube size calculations were recorded.

Results. A total of 61 patients, meanage of $12 \pm 4.21$ (217) years and mean weight of $38 \pm 22.94(10-106) \mathrm{kg}$ wereenrolled. Thediameter of tracheameasured by ultsonography was 13.0(11.4-15.1). Outer diameter $(\mathrm{mm})$ of the ETT determined by anesthesiologist was $8.42 \pm 1.43$; calculated by Cole formula was $9.0 \pm 1,42$; calculated by Khine formula was $7.67 \pm 1.46$; calculated by Motoyama formula was $8.33 \pm 1.42$. ETT cuff was inflated after ETT placement due to leakin $31(47.7 \%)$ patients. Tube was replaced by a larger tube due to excessive leakin one patient. Poor intraclass correlation was found between ultrasonographically determined tracheal diameter and aged based tube diameter calculations (tracheal diameter vs Cole [0.273], Khine [0.207], and Motoyama [0.230]).

Conclusion. Ultrasonographical determination of transverse tracheal diameter is a suitable method for determining the correctendotracheal tube size when compared with the age based formulas. Key words: Child; endotracheal intubation; trachea; ultrasonography.
\end{abstract}

http:/ / dx.doi.org/10.5546/ aap.2018.eng.172

To cite: Gollu G, Bermede AO, Khanmammadov F, et al. Use of ultrasonography as a noninvasive decisive tool to determine the accurate endotracheal tube size in anesthetized children. Arch Argent Pediatr 2018;116(3):172-178.

\section{INTRODUCTION}

In children, determining the correct endotracheal tube (ETT) size is a challenging task. ${ }^{1}$ In daily routine, aged related formulas are commonly used to determine a correct tube size, although such formulas may not be suitable for every child. ${ }^{1-3} \mathrm{~A}$ formula estimated ETT that may be small in size may not provide sufficient ventilatory support, escalates airway resistance, leads inadequate $\mathrm{CO}_{2}$ monitorization, increases the risk of contamination of the operating room with the anesthetic agent and enhances the aspiration risk. ${ }^{1,2,4,5} \mathrm{On}$ the other hand, upper airway injury may occur in the form of ulceration, ischemia, and scarring that may lead subglottic stenosis even after short intubations when larger in size ETT was chosen. ${ }^{1,2,4-6}$ Appropriate estimation and correct sizing of ETT should be the upmost priority of the clinicians for a successful airway management in clinical scenarios.

Recent studies reveal that ultrasonography might be a reliable technique to assess the transverse diameter of trachea. Even though, there are number of adult studies for determination of the correct ETT size, in pediatric population more studies are needed to evaluate the clinical validation of ultrasound for airway management and correlation of it with conventional estimation techniques. ${ }^{1,2,5}$

The aim of this study was to determine tracheal diameter by ultrasonography in children and the correlation with the aged based ETT size estimation formulas. 


\section{PATIENTS AND METHODS}

This prospective study was approved by the local Ethics Committee (Ankara University, School Medicine Ethics Committee, Head, Prof. Dr. Mehmet Melli, Registration 09.09.2014\#, 17743-14, 27.10.2014) and performed in accordance with the ethical standards outlined in the current version of the Declaration of Helsinki and written informed consent. Patients with ASA I-II physical status, who were scheduled to undergo surgery requiring general anesthesia between January 2015 and June 2015 were enrolled to the study. Exclusion criteria were any facial or respiratory tract anomalies, failure to give informed consent, anticipated difficult airway, and having a history of ultrasound gel allergy. All intubations were performed by the same senior anesthesiologist who was blinded to the results of the ultrasonographic examination.

All patients were premedicated with oral $0.5 \mathrm{mg} / \mathrm{kg}$ midazolam. Patients without a vascular access, anesthesia induction was achieved by $8 \%$ sevoflurane in $50 \% \mathrm{O}_{2} / \mathrm{NO}_{2}$ mixture. As soon as vascular access was achieved $0.6-1.2 \mathrm{mg} / \mathrm{kg}$ rocuronium bromide was administrated. If vascular access was available at the admission of the patient to operating room,2-3 mg/ kg intravenous propofol and 0.6$1.2 \mathrm{mg} / \mathrm{kg}$ rocuronium bromide were used to achieve anesthesia induction. Subglottic tracheal area was measured by ultrasonography (Siemens, Acuson X150, 13-5 Hz lineer probe, CA, USA) on $90^{\circ}$ transverse plane when airway pressure was 0 mbar during mask ventilation (at expirium) and the head is in neutral position (Figure 1). The measurements were done by the same surgeon who was trained by an experienced radiologist for two-weeks for ultrasonographic tracheal diameter measurement technique (Figure 2). The surgeon stood on the right side of the patient and the ultrasonography device was placed to the left. The monitor of the ultrasonography device was turned towards the surgeon so that the anesthesiologist would not be able to see it (Figure 2a). Ultrasonography gel was applied to patient's neck and the transducer was transversely placed to the neck in order to measure the subglottic area immediately beneath vocal cords on transverse plane. The anesthesiologist, who was blind to the images and measurements on ultrasound device, determined

FIGURE 1. The settling of ultrasound device, the surgeon and the anesthesiologist

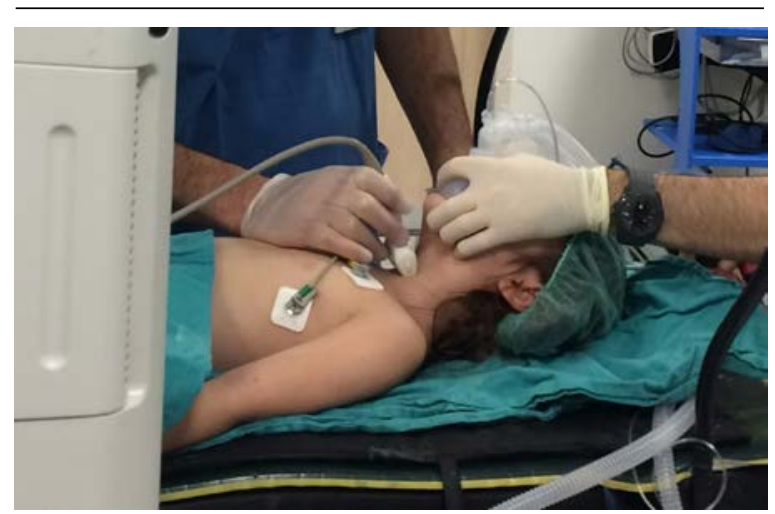

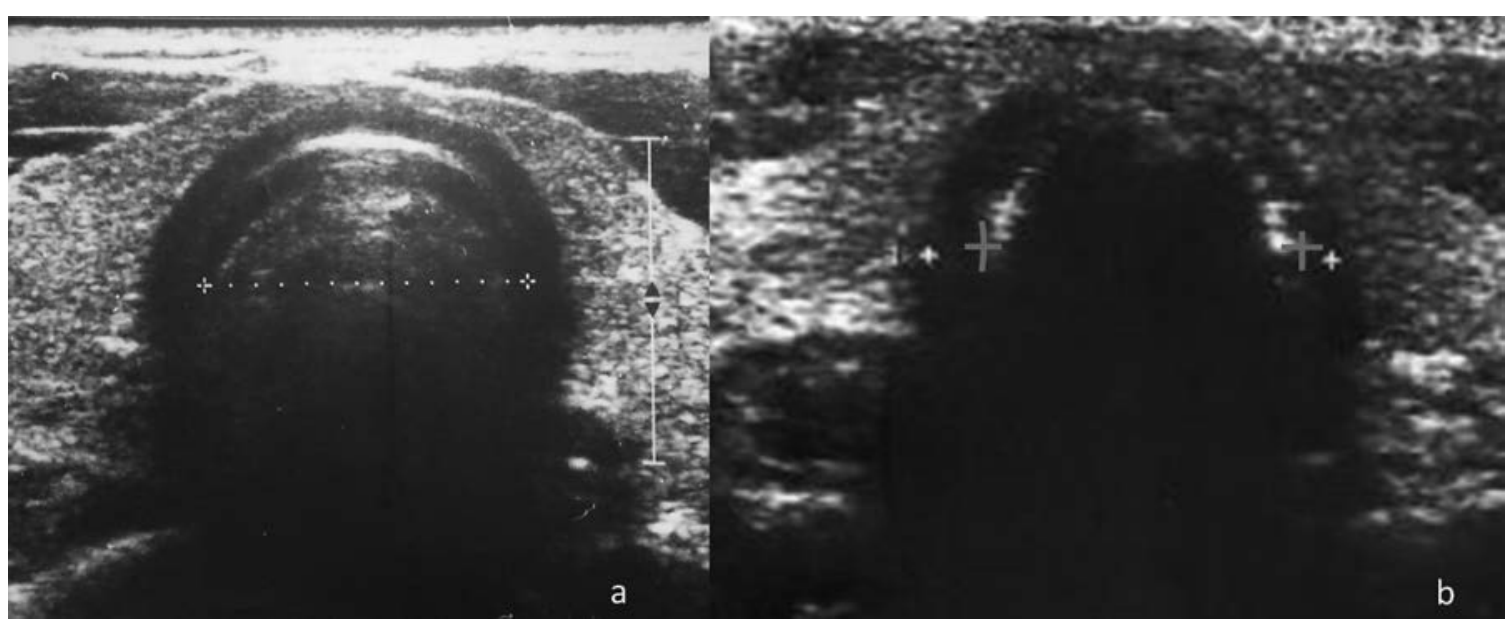

2a. Transverse tracheal diameter measurement with ultrasonography

2b. Ultrasonographic control of ETT placement 
the tube size and performed intubation by evaluating the space between vocal cords with the help of a direct laryngoscopic view. Cuffed tubes (Kaishou, Jiangsu, China) were used in all procedures. Intratracheal ETT position was verified ultrasonographically after the procedure (Figure 2b). As secondary methods of verification, end-tidal $\mathrm{CO}_{2}$ trace, numeric $\mathrm{CO}_{2}$ levels and bilateral respiratory sounds were checked by the anesthesiologist. After the placement of ETT, exhalation valve was set at $20-30 \mathrm{cmH}_{2} \mathrm{O}$, a stethoscope was placed to mouth to check for leak with the head in neutral position. The procedure was considered satisfactory when the leak was insignificant whereas the absence of any leak lead the anesthesiologist think that the tube was too large in size, prompting the anesthesiologist to replace it by a smaller tube. In case of excessive leak, ETT's cuff was inflated with the least cuff volume to prevent cuff leak. If the leakage continued at $20-30 \mathrm{~cm} \mathrm{H}_{2} \mathrm{O}$ inflation pressure, ETT was considered smaller than the accurate one, then cuffed tube sized up by onehalf size. The same procedures were repeated with the replaced ETT. After the procedure, tube calculations were done by ultrasonographically measured diameter; tube's inner and outer diameter; leak/pressure check; and appropriate
ETT size according to the child's age by Cole [ID $(\mathrm{mm})=0.25 \times$ (age in years $)+4]$, Motoyama [ID $(\mathrm{mm})=0.25 \times$ (age in years $)+3.5]$, and Khine $[\mathrm{ID}(\mathrm{mm})=0.25 \times($ age in years $)+3]$ formulas., ${ }^{2,-9}$

\section{Statistical Analysis}

Data analysis was performed in SPSS for Windows 15 software package. The descriptive statistics included mean \pm standard deviation for variables with normal distribution; median (minimum-maximum) for variables with nonnormal distribution; and number and percentage (\%) for nominal variables. The differences between the means and medians were compared using Student's t test and Mann Whitney-U test, respectively. Nominal variables were compared with Pearson's Chi-square test or Fisher's exact test.

The agreement between the two methods was calculated using intra-class correlation agreement coefficient. Bland Altman method was used to calculate measurement differences and their 95\% confidence intervals; the results were shown on Bland Altman graphics. The correlation between continuous variables was tested by Spearman's correlation analysis for non-normally distributed variables and by Pearson's correlation test for normally distributed variables. The significance level was set at $\mathrm{p}<0.05$.

FIGURE 3. The flowchart diagram of patients

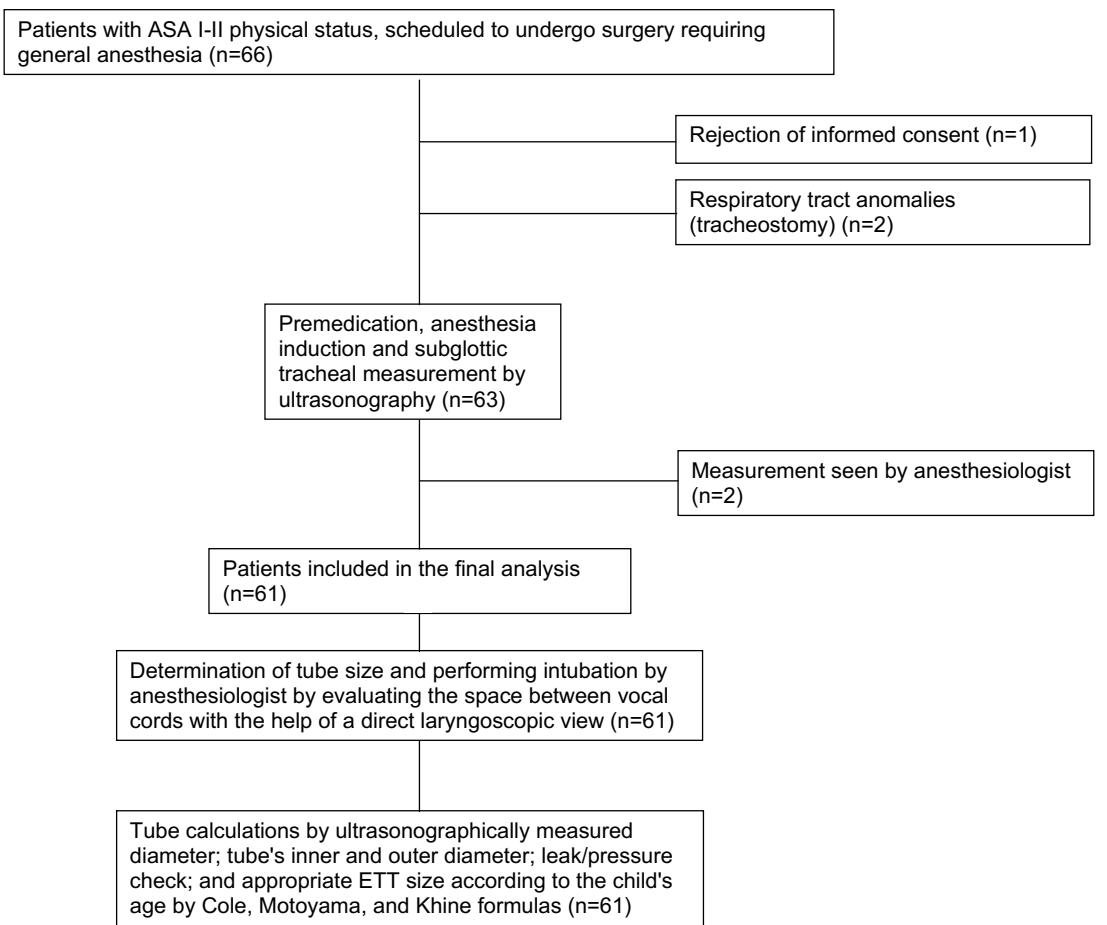




\section{RESULTS}

The flowchart diagram of included patients is shown in Figure 3. Demographic data and type of surgical interventions of the patients enrolled to the study are presented in Table 1. Among the children aged 2-17 years (median $12 \pm 4.21$ years), 35 were male and 26 were female. The weight range of the children was $10-106 \mathrm{~kg}$ (median $38 \pm 22.94 \mathrm{~kg}$ ). The children underwent general anesthesia for acute appendicitis $(n=34)$, perforated appendicitis $(n=6)$, cholelithiasis $(n=2)$, ovarian cyst $(n=2)$, and other indications $(\mathrm{n}=17)$. A laparoscopic intervention was applied in $47(77 \%)$ children.

The smallest and largest inner diameter of the ETTs used for airway management was $4 \mathrm{~mm}$ and $8.5 \mathrm{~mm}$ respectively, with a median inner diameter of $6.5 \mathrm{~mm}$. Following the placement of ETT, cuff was inflated upon the detection of leak in $31(47.7 \%)$ patients. The tube was replaced with a larger one in only one patient due to excessive leak at the $30 \mathrm{cmH}_{2} \mathrm{O}$ cuff pressure. None of the signs of upper respiratory tract obstruction was observed in any patient during the recovery phase of anesthesia or after discharge from the hospital. No accidental esophageal intubation was observed in any patient.

TABLE 1. Demographic data and type of surgical interventions of the patients. Median (min-max) and number of patients

\begin{tabular}{lc}
\hline Number of patients & 61 \\
Age (years) & $12 \pm 4.21(2-17)$ \\
Gender (F/M) (n) & $26 / 35$ \\
Body weight $(\mathrm{kg})$ & $38 \pm 22.94(10-106)$ \\
Type of surgical interventions (n) & \\
Acute appendicitis & 34 \\
Perforated appendicitis & 6 \\
Cholelithiasis & 2 \\
Ovarian cyst & 2 \\
Other & 17 \\
Type of surgery (n) & \\
Laparoscopic & 47 \\
Open/non laparoscopic & 14 \\
\hline
\end{tabular}

The diameter of trachea measured by ultrasound, the outer diameters of endotracheal tube determined by the anesthesiologist and age based formulas were shown in Table 2. The ultrasonographically determined transverse diameter and the tube diameter determined by the anesthesiologist were compared, and an agreement coefficient of 0.273 was found (F value 3.537) $(\mathrm{p}=0.001)$ (Figure $4 a)$. The ICC agreement of the transverse diameters determined by the Cole formula and ultrasonography was 0.273 (F value 2.10) $(\mathrm{p}=0.001)$ (Figure $4 b)$. There was a significant difference between ultrasonographically determined tracheal diameter and the one determined by the Khine formula $(p=0.001)$, with the ICC agreement coefficient being 0.207 (F value 2.957) (Figure 4c). The ICC agreement coefficient of the ultrasonographically determined transverse diameter and the one determined by the Motoyama formula was 0.230 (F value 2.787) $(\mathrm{p}=0.001)$ (Figure $4 d)$. No significant difference was found between the diameters determined by the available formulas and the anesthesiologist $(\mathrm{p}>0.05)$.

\section{DISCUSSION}

This study provides a comparison of an ultrasonographically determined transverse tracheal diameter at subglottic area with one determined by an anesthesiologist and by available formulas. A significant difference was found between the ultrasonographically determined tracheal diameter and the ones determined by the anesthesiologist and by available formulas.

In pediatric patient population, it is not always easy to determine the appropriate ETT size. Variability in tube size may be due to the operator's experience and disproportionate spread of possibility of age and body weight of children. Formulas and methods to determine ETT size can be used to avoid repeat procedures

TABLE 2. The diameter of trachea measured by ultrasound, the outer diameters of endotracheal tube determined by the anesthesiologist and age based formulas (Cole, Khine and Motoyama)

\begin{tabular}{lcc}
\hline & Median, [Percentiles (10-90)] & Mean \pm SD \\
\hline Diameter of trachea (ultrasound assisted) $(\mathrm{mm})$ & $13.0,[11.4-15.1]$ & $13.57 \pm 2.89$ \\
Outer diameter of the endotracheal tube $(\mathrm{mm})$ & & $8.42 \pm 1.43$ \\
Determined by Anesthesiologist & $8.7,[7.3-9.3]$ & $9.0 \pm 1.42$ \\
Calculated by Cole Formula & $9.3,[8.0-10.1]$ & $7.67 \pm 1.46$ \\
Calculated by Khine Formula & $8.0,[6.7-9.0]$ & $8.33 \pm 1.42$ \\
Calculated by Motoyama Formula & $8.7,[7.3-9.4]$ & \\
\hline
\end{tabular}

SD: standard deviation. 
and to facilitate the ETT placement procedure. ${ }^{10,11}$ In this study, all intubations were performed by a well experienced anesthesiologist in the field of pediatric anesthesiology with the guidance of the vocal cord visualization. However, it might be a challenging decision for an inexperienced anesthesiologist to determine the ETT size by just observing the periglottic area. Apparently, the age based formulas were commonly used by most of the anesthesiologists in many centers as it is believed that it might provide an objective solution to a clinical problem. ${ }^{1,4}$ Moreover, in this current study we have demonstrated a significant difference between the ultrasound assisted tracheal diameter, outer diameters of ETTs determined by anesthesiologists and various age based formulas. This clinical difference necessitates a more precise and organized method for determining the accurate tube size. The results of our study reveals that ultrasound can be an objective technique for the clinicians for the assessment of the tracheal diameter and this confirmation may help the clinician to estimate the correct and more suitable ETT size for every single patient.
Studies have shown that the transverse diameter determined at the subglottic area is narrower than the anteroposterior diameter, with the measurement of transverse diameter being preferred owing both to its convenience of measurement and narrowness. ${ }^{6}$

Laryngeal calcification starts above the age of 30 years. As laryngeal calcification is an unexpected finding in children, ultrasonographic measurement had no limitation originated from calcification in this population. ${ }^{6}$

Schramm et al. reported that ultrasonographic measurement of transverse diameter of subglottic area was associated with reduced repeated intubation procedures compared to that determined by the age-based formulas in children younger than 5 years of age. ${ }^{1}$ Furthermore, it has been shown that ultrasonographic measurement and age-based formulas derive an appropriate tube size at a rate of $48 \%$, while other studies reported rates ranging between $47 \%$ and $77 \% .^{1,2}$ Some authors have reported that subglottic diameter can be measured by chest X-Ray, ultrasonography, and magnetic resonance imaging. ${ }^{2-4,6}$ Shibasaki et al., similar to the present

FIGURE 4. Intra-class correlation agreement coefficient between methods
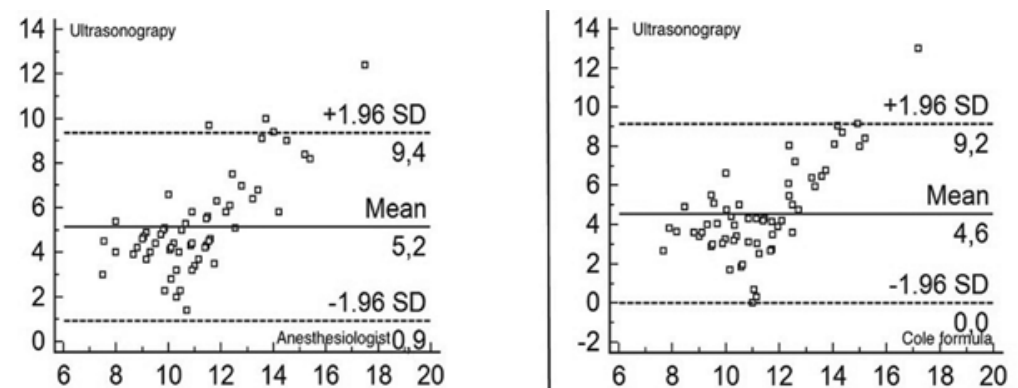

$4 a$

$4 b$

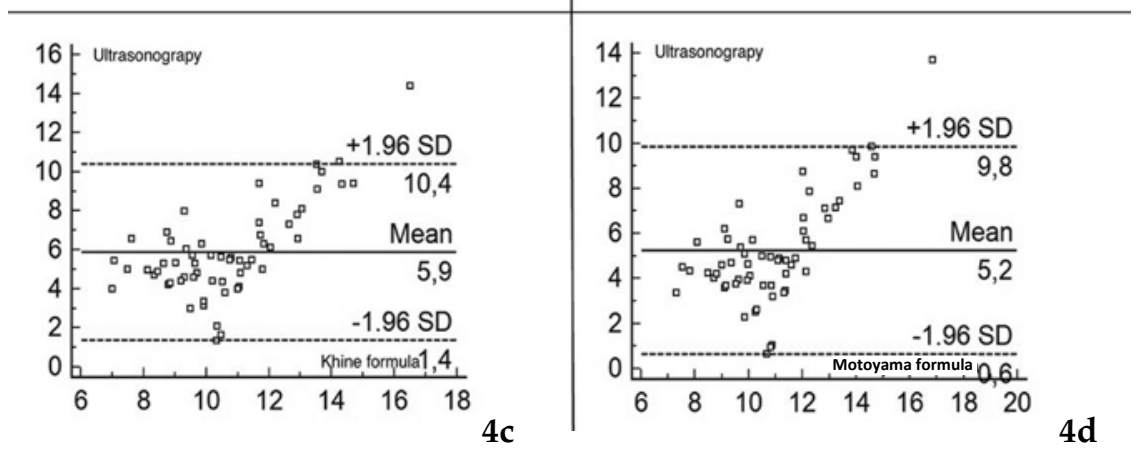

4a: Comparison between ultrasonographically measured transverse tracheal diameter and the anesthesiologists' measurement. 4b: Comparison between ultrasonographically measured transverse tracheal diameter and Cole formula. 4c: Comparison between ultrasonographically measured transverse tracheal diameter and Khine formula. 4d: Comparison between ultrasonographically measured transverse tracheal diameter and Motoyama formula. 
study, demonstrated that ultrasonography was a good predictor in this context. ${ }^{2}$

The limitation of the present study is that the majority of the operated subjects were intubated for brief periods (mean 40 minutes) and all patients were extubated after surgery. Therefore, the anesthesiologist may have preferred small ETTs to avoid postoperative edema and croup development. However, there was no significant difference between the diameters derived from the formulas and the anesthesiologist's measurements. Another limitation of the study is that determining transverse tracheal diameter and verification of tube's position between vocal cords by ultrasonography require a brief training period. Additionally, in this study the appropriate ETT size which is determined by ultrasonographic evaluation are not used in patients so we cannot comment about to leakage or postoperative airway problems when determining ETT size via ultrasonographic evaluation.

\section{CONCLUSION}

The use of ultrasonography is associated with the determination of a more appropriate ETT size, which leads to reduce tube exchange procedures compared to that achieved by anesthesiologists and the available formulas. This non-invasive assessment would be beneficial for children requiring intubation.

Ultrasonographical determination of transverse tracheal diameter is a suitable method for determining the correct endotracheal tube size when compared with the age based formulas.

\section{REFERENCES}

1. Schramm C, Knop J, Jensen $\mathrm{K}$, et al. Role of ultrasound compared to age- related formulas for uncuffed endotracheal intubation in a pediatric population. Paediatr Anaesth 2012;22(8):781-6.

2. Shibasaki M, Nakajima Y, IshiiS, etal.Prediction of pediatric endotracheal tube size by ultrasonography. Anesthesiology 2010;113(4):819-24.

3. Hardee PS, Ng SY, Cashman M. Ultrasound imaging in the preoperative estimation of the size of tracheostomy tube required in specialize doperations in children. $\mathrm{Br} \mathrm{J}$ Oral Maxillofac Surg 2003;41(5):312-6.

4. Gupta K, Gupta PK, Rastogi B, et al. Assessment of the subglottic region by ultrasonography for estimation of appropriate size endotracheal tube: A clinical prospective study. Anesth Essays Res 2010;6(2):157-60.

5. Orf J, Thomas SH, Ahmed W, et al. Appropriateness of endotracheal tube size and insertion depth in children undergoing air medical transport. Pediatr Emerg Care 2000;16(5):321-7.

6. Lakhal K, Delplace X, Cottier JP, et al. The feasibility of ultrasound to assess subglottic diameter. Anesth Analg 2007:104(3):611-4.

7. Khine HH, Corddry DH, Kettrick RG, et al. Comparison of cuffed and uncuffed endotracheal tubes in young children during general anesthesia. Anesthesiology 1997;86(3):62731.

8. Cole F. Pediatric formulas for the anesthesiologist. $A M A$ J Dis Child. 1957;94(6):672-3.

9. Motoyama EK. Endotracheal intubation. In:Motoyama EK, Davis PJ, eds, Smith's Anesthesia for infants and children. $5^{\text {th }}$ ed. St Louis: Mosby; 1990.p.269-75.

10. Chou HC, Tseng WP, Wang $\mathrm{CH}$, et al. Tracheal rapid ultrasound exam (T.R.U.E.) for confirming endotracheal tube placement during emergency intubation. Resuscitation 2011;82(10):1279-84.

11. Göksu E, Sayrac V, Oktay C, et al. How to stylet use can effect confirmation of endotracheal tube position using ultrasound. Am J Emerg Med 2010;28(1):32-6. 